CERN-TH/96-49

hep-th/9602096

\title{
BIRTH OF THE UNIVERSE AS QUANTUM SCATTERING IN STRING COSMOLOGY
}

\author{
M. Gasperini円 and G. Veneziano \\ Theory Division, CERN, CH-1211 Geneva 23, Switzerland
}

\begin{abstract}
In a Wheeler-De Witt approach to quantum string cosmology, the present state of the Universe arises from the scattering and reflection of the wave function representing the initial string vacuum in superspace. This scenario is described and compared with the more conventional quantum cosmology picture, in which the birth of the Universe is represented as a process of tunnelling "from nothing" in superspace.
\end{abstract}

Essay written for the 1996 Awards of the Gravity Research Foundation (Wellesley Hills, Ma, 02181-0004)

CERN-TH/96-49

February 1996

\footnotetext{
${ }^{1}$ Permanent address: Dipartimento di Fisica Teorica, Via P. Giuria 1, 10125 Turin, Italy.
} 
In the standard cosmological model, the birth of our Universe is assumed to coincide with the initial big-bang singularity, characterizing the classical solutions of the Einstein cosmological equations. Near the singularity, however, the Universe approaches the Planck curvature scale and the quantum gravity regime, where a classical description of the spacetime manifold is no longer appropriate. By adopting, in that regime, a quantum cosmology approach, one can describe the birth of the Universe as a "tunnelling from nothing" [1]-[3], where the process of tunnelling refers to the Wheeler-De-Witt (WDW) wave function [4] in superspace.

In string cosmology models [5], the Universe starts evolving from the string perturbative vacuum, a state with flat metric and vanishing coupling constant. The curvature and the coupling grow during an initial "pre-big-bang" phase, and this growth, according to the low-energy effective action, leads classically to a singular state which marks the beginning of the standard, post-big-bang cosmological era. By applying a quantum cosmology approach, the transition through the singular big-bang regime can be described as a scattering of the initial pre-big-bang state into a final post-big-bang configuration, in particular as a reflection of the WDW wave function in superspace. The purpose of this paper is to illustrate this effect and to stress analogies and differences with the more conventional tunnelling scenario for the birth of the Universe.

For an easier comparison of the two pictures we shall work with the simplest example of non-trivial WDW equation, in which the effective potential is the one induced by a positive cosmological constant $\Lambda$, due to an over-critical number of dimensions. We start with the tree-level, low-energy string effective action [6]

$$
S=-\frac{1}{2 \lambda_{s}^{2}} \int d^{4} x \sqrt{-g} e^{-\phi}\left(R+\partial_{\mu} \phi \partial^{\mu} \phi+\Lambda\right),
$$

where $\phi$ is the dilaton field, $\lambda_{s}$ is the fundamental string-length parameter governing the derivative expansion of the effective action, and the extra dimensions have been taken to be completely inert. For a homogeneous, isotropic and spatially flat metric background, with scale factor $a$ and spatial sections of finite volume, we define:

$$
\bar{\phi}=\phi-\ln \int\left(d^{3} x / \lambda_{s}^{3}\right)-\sqrt{3} \beta, \quad \beta=\sqrt{3} \ln a .
$$

In the cosmic-time gauge, $g_{00}=1$, the action (10) becomes:

$$
S=-\frac{\lambda_{s}}{2} \int d t e^{-\bar{\phi}}\left(\dot{\bar{\phi}}^{2}-\dot{\beta}^{2}+\Lambda\right) .
$$


By using the convenient time reparameterization $d t=d \tau e^{-\bar{\phi}}$ we are finally led to the Hamiltonian

$$
H=\frac{1}{2 \lambda_{s}}\left(\Pi_{\beta}^{2}-\Pi_{\phi}^{2}+\lambda_{s}^{2} \Lambda e^{-2 \bar{\phi}}\right)
$$

where $\Pi_{\beta}, \Pi_{\bar{\phi}}$ are the canonical momenta

$$
\Pi_{\beta}=\frac{\delta S}{\delta \beta^{\prime}}=\lambda_{s} \beta^{\prime}, \quad \Pi_{\bar{\phi}}=\frac{\delta S}{\delta \bar{\phi}^{\prime}}=-\lambda_{s} \bar{\phi}^{\prime},
$$

and a prime denotes differentiation with respect to $\tau$.

This Hamiltonian implies momentum conservation along the $\beta$ axis,

$$
\left[\Pi_{\beta}, H\right]=0, \quad \Pi_{\beta}=\lambda_{s} \dot{\beta} e^{-\bar{\phi}}=k=\text { const. }
$$

The general solution of the classical equations of motion is well known [7], [8] and contains two distinct branches,

$$
a(t)=a_{0}(\tanh |\sqrt{\Lambda} t / 2|)^{ \pm 1 / \sqrt{3}}, \quad \bar{\phi}=\phi_{0}-\ln \sinh |\sqrt{\Lambda} t|, \quad k= \pm \lambda_{s} \sqrt{\Lambda} e^{-\phi_{0}}
$$

where $a_{0}$ and $\phi_{0}$ are integration constants. The two branches are defined over disconnected ranges of time, $t<0$ and $t>0$, separated by a singularity of the curvature invariants and of the effective string coupling $e^{\bar{\phi}}$ at $t=0$ (for simplicity, we have chosen the integration constants so as to make the singular ends of both time ranges coincide at $t=0$ ).

We are interested, in particular, in the branch describing a classical approach to the singularity in a state of accelerated expansion, growing curvature, typical of the pre-bigbang regime [5]

$$
\begin{aligned}
t<0, \quad a & =a_{0}[\tanh (-\sqrt{\Lambda} t / 2)]^{-1 / \sqrt{3}}, \quad \bar{\phi}-\phi_{0}=-\ln \sinh (-\sqrt{\Lambda} t) \\
\dot{a} & >0, \quad \ddot{a}>0, \quad \dot{H}>0, \quad k=\lambda_{s} \sqrt{\Lambda} e^{-\phi_{0}}>0
\end{aligned}
$$

and in the branch emerging from the singularity in a state of decelerated expansion, decreasing curvature,

$$
\begin{array}{rlrl}
t>0, & a & =a_{0}[\tanh (\sqrt{\Lambda} t / 2)]^{1 / \sqrt{3}}, & \bar{\phi}-\phi_{0}=-\ln \sinh (\sqrt{\Lambda} t) \\
\dot{a} & >0, \quad \ddot{a}<0, \quad \dot{H}<0, \quad & k=\lambda_{s} \sqrt{\Lambda} e^{-\phi_{0}}>0 .
\end{array}
$$

Both branches have positive canonical momentum $\Pi_{\beta}=k>0$, and are related by a duality transformation including time-reflection [5, 8], $a(t) \rightarrow a^{-1}(-t), \bar{\phi}(t) \rightarrow \bar{\phi}(-t)$. Let us call, 
respectively, (+) and $(-)$ the pre- and post-big-bang branches (8) and (9). In the highcurvature, strong coupling regime $\bar{\phi} \rightarrow+\infty$ (i.e. near the singularity) they are characterized by a constant and opposite value of the canonical momentum along $\bar{\phi}$, namely

$$
\lim _{\bar{\phi} \rightarrow+\infty} \Pi_{\bar{\phi}}^{( \pm)}=\lim _{\bar{\phi} \rightarrow+\infty}\left(-\lambda_{s} \dot{\bar{\phi}} e^{-\bar{\phi}}\right)_{ \pm}=\mp k .
$$

In the low-energy limit, $\bar{\phi} \rightarrow-\infty$, the two branches still have opposite canonical momentum $\Pi_{\bar{\phi}}$. The momentum is no longer constant, however, but controlled by $\bar{\phi}$,

$$
\lim _{\bar{\phi} \rightarrow-\infty} \prod_{\bar{\phi}}^{( \pm)} \sim \mp \lambda_{s} \sqrt{\Lambda} e^{-\bar{\phi}}
$$

We shall now apply the WDW equation [4], $H \Psi=0$, to compute the (classically forbidden) probability of transition from one branch to another, assuming in particular as the initial state the pre-big-bang configuration represented classically by the solution (8). For the Hamiltonian (4), the WDW equation is a simple differential equation in the two-dimensional minisuperspace parameterized by $\bar{\phi}$ and $\beta$,

$$
\left(\partial_{\bar{\phi}}^{2}-\partial_{\beta}^{2}+\lambda_{s}^{2} \Lambda e^{-2 \bar{\phi}}\right) \Psi(\bar{\phi}, \beta)=0
$$

(there is no problem of operator ordering, as the order is uniquely fixed by the duality symmetries of the string effective action [9]). By exploiting the conservation property (6) we impose

$$
\Pi_{\beta} \Psi_{k}=i \partial_{\beta} \Psi_{k}=k \Psi_{k}
$$

(note the role of the time-like coordinate assigned to $\beta$, which is monotonically increasing from $-\infty$ to $+\infty$ ), and eq. (12) can be separated by setting

$$
\Psi_{k}(\bar{\phi}, \beta)=\psi_{k}(\bar{\phi}) e^{-i k \beta}, \quad\left(\partial_{\bar{\phi}}^{2}+k^{2}+\lambda_{s}^{2} \Lambda e^{-2 \bar{\phi}}\right) \psi_{k}(\bar{\phi})=0
$$

The general solution of eq. (14) is a linear combination of Bessel functions [10, $J_{\nu}(z)$ and $J_{-\nu}(z)$, of index $\nu=i k$ and argument $z=\lambda_{s} \sqrt{\Lambda} e^{-\bar{\phi}}$. In order to fix the boundary conditions we observe that, in the strong coupling regime $\bar{\phi} \rightarrow+\infty$, the effective potential of eq. (14) becomes negligible, and the WDW solution can be written in the plane wave form as

$$
\Psi_{+\infty}^{( \pm)}(\bar{\phi}, \beta)=\lim _{z \rightarrow 0} J_{ \pm i k}(z) e^{-i k \beta} \sim e^{-i k(\beta \mp \bar{\phi})}
$$


In this limit, right- and left-moving waves along $\bar{\phi}$ correspond, respectively, to the pre- and post-big-bang classical configurations (8) and (9). Indeed,

$$
\Pi_{\bar{\phi}} \Psi_{+\infty}^{( \pm)}=i \partial_{\bar{\phi}} \Psi_{+\infty}^{( \pm)}=\mp k \Psi_{+\infty}^{( \pm)}
$$

which is the quantum analogue of the classical relation (10) (the opposite sign with respect to standard conventions, in the differential representation of $\Pi_{\bar{\phi}}$, is due to the negative sign appearing in the definition (5)). Consistently with the chosen pre-big-bang initial conditions, we thus impose that there are only right-moving waves approaching the singularity at $\bar{\phi} \rightarrow$ $+\infty$. This is the same as imposing tunnelling boundary conditions [3], which select only outgoing modes at the (singular) boundary of superspace, and uniquely fixes the WDW wave function as $\left(N_{k}\right.$ is a normalization factor)

$$
\Psi_{k}(\bar{\phi}, \beta)=N_{k} J_{-i k}\left(\lambda_{s} \sqrt{\Lambda} e^{-\bar{\phi}}\right) e^{-i k \beta}
$$

In the low-energy limit $\bar{\phi} \rightarrow-\infty, z \rightarrow \infty$, the effective potential induced by $\Lambda$ becomes dominant, but the solution can still be separated into a left- and a right-moving part according to the asymptotic behaviour of $J_{-\nu}(z)$, namely [10]

$$
\begin{aligned}
\lim _{\bar{\phi} \rightarrow-\infty} \Psi_{k}(\bar{\phi}, \beta) & \sim \Psi_{-\infty}^{(+)}+\Psi_{-\infty}^{(-)} \\
\Psi_{-\infty}^{( \pm)}(z, \beta) & =N_{k}\left(\frac{1}{2 \pi z}\right)^{1 / 2} \exp \left[-i(k \beta \pm z) \pm \frac{i \pi}{4} \pm \frac{k \pi}{2}\right], \quad z=\lambda_{s} \sqrt{\Lambda} e^{-\bar{\phi}}
\end{aligned}
$$

By applying the momentum operator we find

$$
\lim _{\bar{\phi} \rightarrow-\infty} \Pi_{\bar{\phi}} \Psi_{-\infty}^{( \pm)}(\bar{\phi}, \beta)=\mp z \Psi_{-\infty}^{( \pm)}(\bar{\phi}, \beta)
$$

so that $\Psi_{-\infty}^{(+)}(\bar{\phi}, \beta)$ and $\Psi_{-\infty}^{(-)}(\bar{\phi}, \beta)$ correspond, respectively, to the pre- and post-big-bang branches of the low-energy classical solution, according to eq. (11). Starting from a pre-bigbang initial state, we thus obtain a finite probability of transition to the "dual" low-energy state. The transition is represented as a reflection of the wave function in minisuperspace [9], and the probability is measured by the reflection coefficient $R_{k}$ as

$$
R_{k}=\frac{\left|\Psi_{-\infty}^{(-)}\right|^{2}}{\left|\Psi_{-\infty}^{(+)}\right|^{2}}=e^{-2 \pi k}
$$


By recalling the definition of $k$ and $\bar{\phi}$, the transition probability for a three-dimensional portion of space of initial proper volume $\Omega_{i}$ at $t \rightarrow-\infty$ can be written as

$$
R\left(\Lambda, \Omega_{i}, g_{s}\right)=\exp \left\{-\frac{\sqrt{12} \pi}{g_{s}^{2}} \frac{\Omega_{i}}{\lambda_{s}^{3}}\left[\frac{\sqrt{3}}{\lambda_{s} \sqrt{\Lambda}}+\left(1+\frac{3}{\lambda_{s}^{2} \Lambda}\right)^{1 / 2}\right]^{\sqrt{3}}\right\}
$$

where $g_{s}=e^{\phi_{s} / 2}$ is the value of the string coupling at the scale $H_{s}=\lambda_{s}^{-1}$. Quite interestingly, this probability is independent of $\Lambda$ for $\Lambda>>\lambda_{s}^{-2}$, and it is peaked in the strong coupling regime with a typical instanton-like dependence on the coupling constant, $R \sim \exp \left(-g_{s}^{-2}\right)$. This probability is also invariant under the T-duality transformation 11$]\left(\Omega_{i} / \lambda_{s}^{3}\right) \rightarrow\left(\lambda_{s}^{3} / \Omega_{i}\right)$, $g_{s}^{2} \rightarrow g_{s}^{2}\left(\Omega_{i} / \lambda_{s}^{3}\right)^{-2}$.

By contrast, the probability of birth of the Universe from quantum tunnelling may be computed from the Einstein-de Sitter action

$$
S=-\frac{1}{2 \lambda_{p}^{2}} \int d^{4} x \sqrt{-g}(R+\Lambda)
$$

where $\lambda_{p}$ is the Planck length. Solving the corresponding WDW equation, with appropriate boundary conditions, the tunnelling probability can be estimated as [1, 2, 3]

$$
P \sim \exp \left\{-\frac{4}{\lambda_{p}^{2} \Lambda}\right\}
$$

The main difference between the above string scenario and the standard one is that, in the latter, the Universe emerges from the quantum era in a classical inflationary regime, and the tunnelling process is completely controlled by the value of the cosmological constant. By contrast, in our case, the quantum era is approached at the end of a classical inflationary epoch. In both cases, however, the appropriate boundary conditions imposed at the big-bang singularity play a crucial role, and the probability of the birth of our Universe is given by the ratio of the (squared modulus of the) final wave function representing a standard decelerated expansion and the initial wave function. Also, in both cases the quantum process seems to favour large values (in Planck or string units) of the cosmological constant.

It is perhaps worth recalling, at this point, the words of Vilenkin while presenting his "tunnelling from nothing" scenario [1]-[3]:

"... Here "nothing" means the vacuum of some more fundamental theory...."

Our work suggests that "nothing" can be just the perturbative vacuum of string theory. The different description of the birth process (a wave reflection, rather than a tunnelling) simply 
originates from the deep differences between string and Einstein gravity, which in the string case allow for a long, classical (and inflationary!) pregnancy. 


\section{References}

[1] A. Vilenkin, Phys. Lett. B117, 25 (1982).

[2] A. Vilenkin, Phys. Rev. D30, 509 (1984).

[3] A. Vilenkin, Phys. Rev. D33, 3650 (1986);

A. Vilenkin, Phys. Rev. D37, 888 (1988).

[4] B. S. De Witt, Phys. Rev. 160, 1113 (1967);

J. A. Wheeler, in Battelle Rencontres, ed. by C. De Witt and J. A. Wheeler (Benjamin, New York, 1968).

[5] M. Gasperini and G. Veneziano, Astropart. Phys. 1, 317 (1993) ;

M. Gasperini and G. Veneziano, Mod. Phys. Lett. A8, 3701 (1993);

M. Gasperini and G. Veneziano, Phys. Rev. D50, 2519 (1994);

R. Brustein and G. Veneziano, Phys. Lett. B329, 429 (1994).

[6] C. Lovelace, Phys. Lett. B135, 75 (1984);

E. S. Fradkin and A. A Tseytlin, Nucl. Phys. B261, 1 (1985);

C. G. Callan et al., Nucl. Phys. B262, 593 (1985).

[7] M. Muller, Nucl. Phys. B337, 37 (1990).

[8] G. Veneziano, Phys. Lett. B265, 387 (1991).

[9] M. Gasperini, J. Maharana and G. Veneziano, Graceful exit in quantum string cosmology, Preprint CERN-TH/96-32 (hep-th/9602078).

[10] M. Abramowicz and I. A. Stegun, Handbook of Mathematical Functions (Dover, New York, 1972).

[11] For a review see A. Giveon, M. Porrati and E. Rabinovici, Phys. Rep. 244, 77 (1994). 